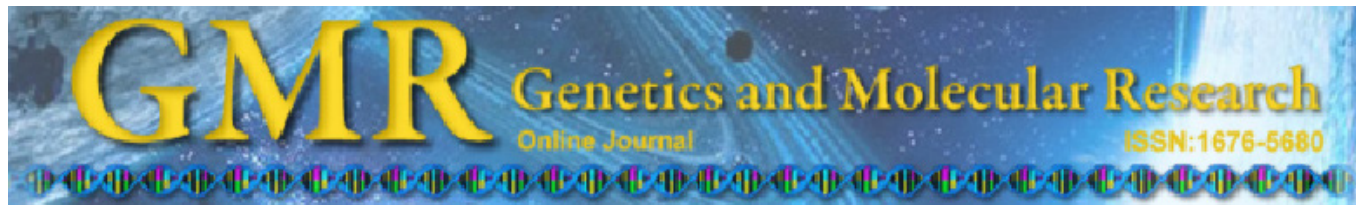

\title{
Effect of lentivirus-mediated RNA interference of APC-Cdh1 expression on spinal cord injury in rats
}

\author{
Y.-H. Qi ${ }^{1,2}$, W.-L. Yao ${ }^{1}$, C.-H. Zhang ${ }^{1}$ and Y.-Q. Guo ${ }^{2}$ \\ 'Department of Anesthesiology, Tongji Hospital, Tongi Medical College, \\ Huazhong University of Science and Technology, Wuhan, China \\ ${ }^{2}$ Department of Anesthesiology, Shanxi Provincial People's Hospital, \\ Taiyuan, China \\ Corresponding authors: Y.-Q. Guo / C.-H. Zhang \\ E-mail: gyq106968@yahoo.com.cn / hqqgen@126.com
}

Genet. Mol. Res. 13 (1): 1366-1372 (2014)

Received January 15, 2013

Accepted July 10, 2013

Published February 28, 2014

DOI http://dx.doi.org/10.4238/2014.February.28.9

\begin{abstract}
This study investigated cadherin-1 (Cdh1) expression in the sensorimotor cortex of rats after spinal cord injury (SCI). The repairing effect of $\mathrm{Cdh} 1$ was evaluated by silencing its expression with lentivirus-mediated RNAi. Twenty male Sprague-Dawley (SD) rats were randomly divided into a normal group and an operation group. Rats of the operation group were given SCI by the Allen method (T10-T11). Cdh1 expression in the sensorimotor cortex was examined by quantitative real-time polymerase chain reaction (PCR) and Western blot analysis. Thirty male SD rats were divided into a sham-operation (SO) group, a lentivirus vector (LV) group, and a recombinant lentivirus (RL) group. Rat behavior was evaluated using the Basso-BeattieBresnahan (BBB) test every week. Ten days after injection, Cdh1 expression was examined by quantitative real-time PCR and Western blot. Six weeks after injury, animals were injected with biotinylated dextran amine-Texas Red (BDA-TR), and then at 8 weeks, spinal cords were removed and sectioned in serial order. The expression of Cdh1
\end{abstract}


mRNA was significantly higher in the operation than in the normal group ( $\mathrm{P}<0.05)$. The expression of Cdh1 mRNA was lower in the $\mathrm{RL}$ than in the SO or LV groups at 10 days after injection $(\mathrm{P}<0.05)$. In addition, the BBB score was higher for the RL than for the SO or LV groups at 6 weeks after injury $(\mathrm{P}<0.05)$. A novel population of BDA-labeled axons was observed extending past the lesion in the RL group, which was rarely observed in the SO and LV groups. These results suggest that the anaphase-promoting complex-Cdh1 may play an important role in inhibiting axonal growth.

Key words: Spinal cord injury; Anaphase promoting complex; Lentivirus; RNAi

\section{INTRODUCTION}

The anaphase-promoting complex (APC) is a multi-subunit complex that acts as a cell cycle-regulated ubiquitin-protein ligase (Vogel, 2004; Wang et al., 2008). Cadherin-1 (Cdh1) is a co-activator protein that confers substrate specificity to the APC. Recent studies have found that Cdh1 and subunits of the APC are expressed in mammalian neurons of the cortex, hippocampus, and cerebellum, and the APC-Cdh1 complex appears to play a role in regulating axonal growth and patterning in the developing brain (Konishi et al., 2004; Stegmüller et al., 2006). In this study, we investigated the expression of Cdh1 in the sensorimotor cortex of rats after spinal cord injury (SCI) and found a functional interaction between the APC and axonal growth. These results extend our understanding of the molecular mechanisms mediating the intracellular signaling of APC-Cdh1 and should lead to a clearer mechanistic view of the inhibition of axonal growth.

\section{MATERIAL AND METHODS}

\section{Animals}

The Institutional Animal Care and Use Committee at the Tongji Medical College of the Huazhong University of Science \& Technology approved all experimental procedures with Sprague-Dawley (SD) rats weighing 200-300 g.

\section{Lentiviral vectors and regents}

Cdh1-siRNA lentiviral vectors were provided and constructed by Shanghai GeneChem Co., Ltd. Western blot analysis was performed using the anti-Cdh1 (Oncogen) and anti-ski-related novel protein N (SnoN; Becton, Dickinson and Company) antibodies. To trace the corticospinal tract, biotinylated dextran amine-Texas Red (BDA-TR) was provided by Vector Laboratories.

\section{SCI model preparation}

Twenty healthy male SD rats were obtained at 11 weeks of age for this study. 
Before the experiments, the rats were bred in our institute at a constant temperature $\left(23^{\circ}\right.$ $\left.\pm 3^{\circ} \mathrm{C}\right), 40-50 \%$ humidity and an indoor noise level less than $60 \mathrm{~dB}$. Rats were randomly divided into a normal group $(\mathrm{N}=10)$ and an operation group $(\mathrm{N}=10)$. The normal group received sham operations of laminectomies only. The operated group was given laminectomies and SCI following the Allen method. Because the rats that underwent SCI lost their ability to micturate, bladder expressions were performed manually until function returned or until the animal was killed. The normal and operation group rats were killed after 1 week and 2 weeks, respectively. The expressions of Cdh1 in the sensorimotor cortex and in injured myeloid tissues were examined by quantitative real-time polymerase chain reaction (PCR). Immunohistochemical staining was further applied using the avidin-biotin complex $(\mathrm{ABC})$ method.

Another 30 male rats were randomly assigned to a sham-operation group ( $\mathrm{SH})$, a lentivirus vector group (LV), and a recombinant lentivirus group (RL). The SH group (N $=8$ ) contained healthy animals that did not undergo injury or injection of any kind. The LV group $(\mathrm{N}=7)$ was given laminectomies and SCIs, followed by $10 \mu \mathrm{L}$ cortical lentiviral vector injections 7 days after surgery. The $R L$ group $(N=7)$ received laminectomies and SCI followed by $10 \mu \mathrm{L}$ cortical recombinant lentiviral vector injections 7 days after surgery. Ten days after injection, the expression of Cdh1 was examined by quantitative real-time PCR.

\section{Quantitative real-time PCR (RT-PCR)}

Total RNA was isolated from sensorimotor cortical tissues using NucleoSpin RNA II (Takara, Japan) according to manufacturer protocols. Reverse transcription was performed with the Revert Aid First-Strand cDNA Synthesis kit (Fermentas, Lithuania), according to manufacturer instructions. Multiplex semi-quantitative RT-PCR was performed using SIP1specific primers (Cdh1). Primer sequences are provided as supplementary information: Cdh1 (XM_001075601) sense primer: 5'-AGCTACCCCAGGACACCCAA-3'; antisense primer: 5'-GCAACGCAATCAGAGTCAACG-3'; (PCR product was 104 bp long); $\beta$-actin (NM_031144) sense primer: 5'-TGACAGGATGCAGAAGGAGA-3'; antisense primer: 5'-TAGAGCCACCAATCCACACA-3'.

Quantitative analysis of the expression of Cdh1 was performed by real-time RT-PCR on cDNAs from the sensorimotor cortical tissues qPCR panel I (OriGene, Rockville, MD, USA), according to manufacturer instructions. The program for the SYBR Green PCR was as follows: 40 cycles at $94^{\circ} \mathrm{C}$ for $15 \mathrm{~s}, 60^{\circ} \mathrm{C}$ for $20 \mathrm{~s}$, relative levels of Cdh1 were measured using a modified $\Delta \mathrm{Ct}$ formula $(\Delta \mathrm{Ct}=\mathrm{Ct}(\mathrm{Cdh} 1)-\mathrm{Ct}(\beta$-actin). Statistical analyses were performed using the Student $t$-test (Schmittgen and Livak, 2008).

\section{Tract tracing}

To trace the corticospinal tract, fluorescent tracers (Fluoro-Gold and Fluoro-Ruby) were injected into the bilateral cortex 14 days before animals were killed to label corticospinal axons. Sections were viewed under fluorescent illumination at 365 and $485 \mathrm{~nm}$ for FluoroGold and Fluoro-Ruby, respectively. The injection sites and lesions were examined in all animals to ensure that there was no unintended diffusion of tracers into the injured spinal cord. 
For BDA-TR injections, mice were anesthetized as described above and placed in a stereotaxic device, the fur was removed by shaving, the scalp was incised, and the skull overlying the sensorimotor cortex was carefully removed with a dental drill. Approximately $10,000 \mathrm{MW}$ Miniruby BDA in $10 \% \mathrm{dH}_{2} \mathrm{O}$ (Invitrogen) was injected into four sites $(1 \mu \mathrm{L}$ per site over a 3-5-min period) using a $10-\mu \mathrm{L}$ Hamilton microsyringe tipped with a pulled glass micropipette. The coordinates of injection sites were 0.5 lateral, $3 \mathrm{~mm}$ deep to the cortical surface, and $-0.5,-1.5 \mathrm{~mm}$ with respect to the bregma. After the injections were completed, the skin overlying the skull was sutured with 4-0 silk. Rat behavior was evaluated by the BassoBeattie-Bresnahan (BBB) assessment every week.

\section{RESULTS}

\section{Expression of Cdh1 mRNA in rats}

The expressions of Cdh1 in the sensorimotor cortex and in injured myeloid tissues were examined by quantitative real-time PCR after SCI. The expression level of Cdh1 mRNA in the operation group was significantly higher than that of the normal group $(\mathrm{P}<0.05)$ (Figure 1). The expression level of Cdh1 mRNA in the RL group was lower than that of the SH group or the LV group 10 days after injection $(\mathrm{P}<0.05$; Figure 2$)$.

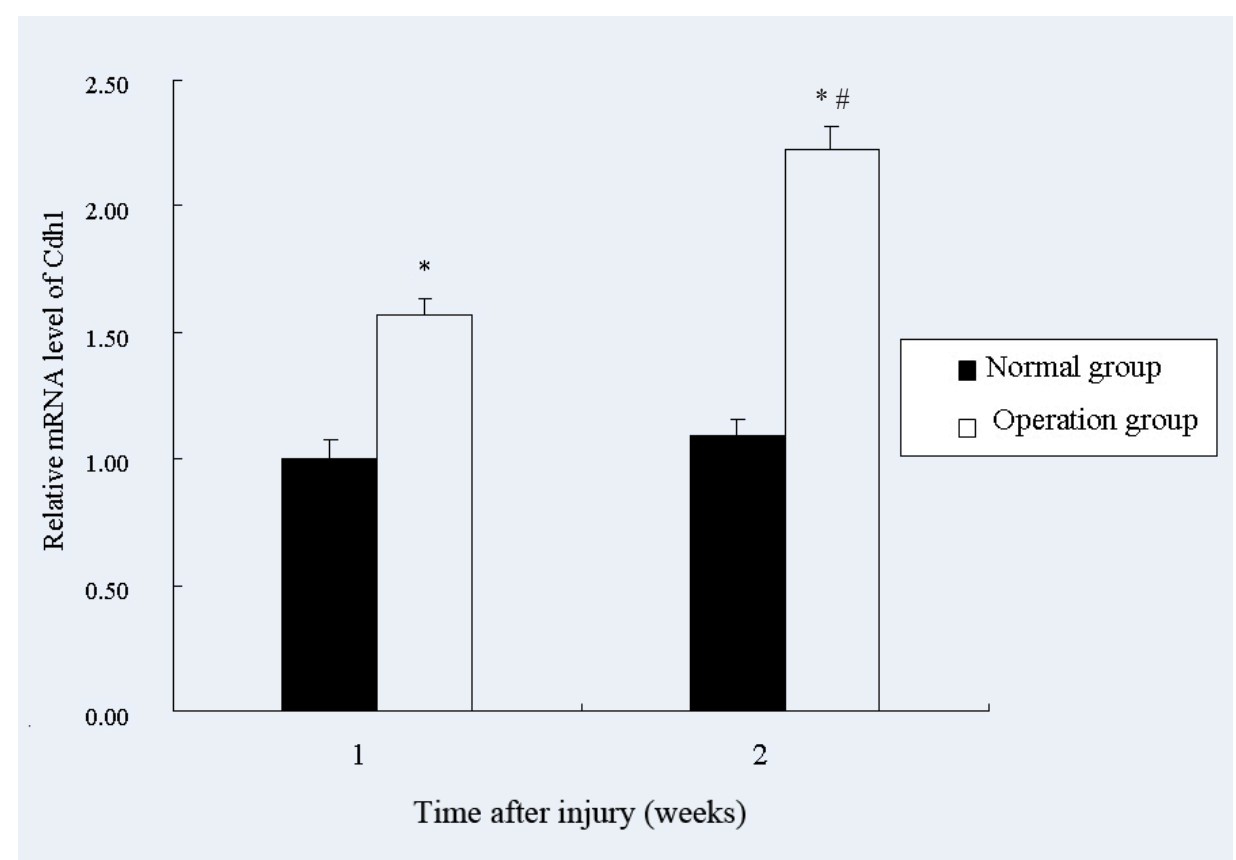

Figure 1. Expression of $\mathrm{Cdh} 1$ in the sensorimotor cortical and injured myeloid tissue was examined by quantitive real-time PCR after spinal cord injury. ${ }^{*} \mathrm{P}<0.05$, compared with control group; ${ }^{*} \mathrm{P}<0.05$, compared with the former one time point. 


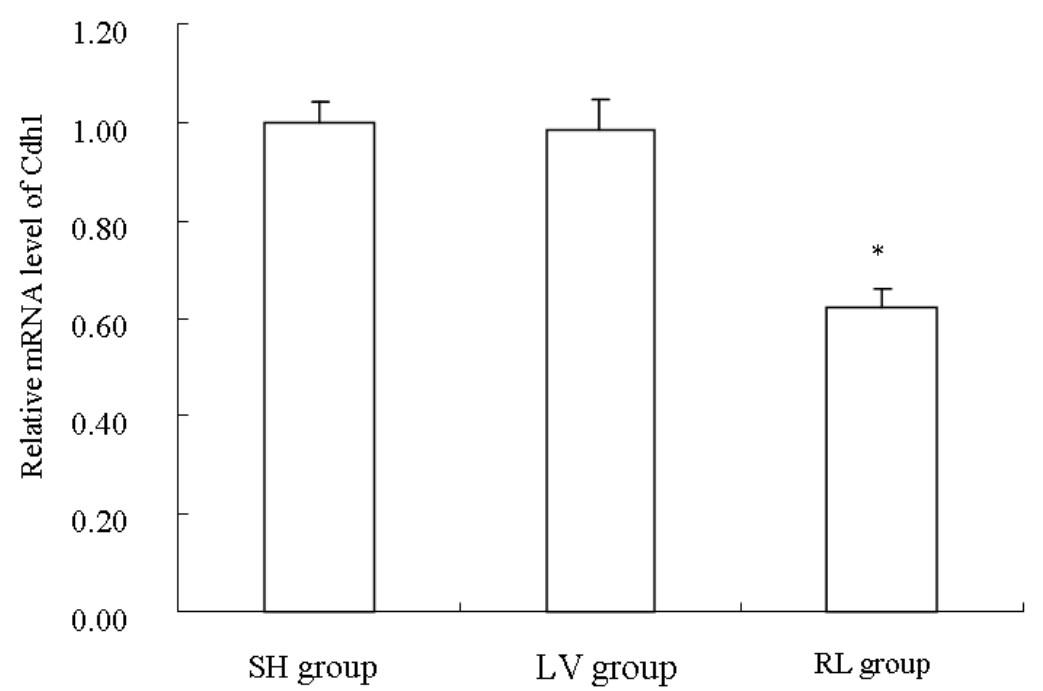

Figure 2. Spinal cord injury followed by cortical lentiviral vector injection 7 days after surgery. Ten days after injection, the expression of Cdh1 was examined by quantitive real-time PCR. SH = sham-operated group; LV = lentivirus vector group; $\mathrm{RL}=$ recombinant lentivirus group. ${ }^{*} \mathrm{P}<0.05$, compared with $\mathrm{SH}$ and $\mathrm{LV}$ groups.

\section{Behavioral analysis}

Rat behavior was evaluated by the BBB test every week after SCI. The BBB score was significantly higher in the RL group compared to the SH or LV groups 6 weeks after injury $(\mathrm{P}<0.05$; Figure 3$)$.

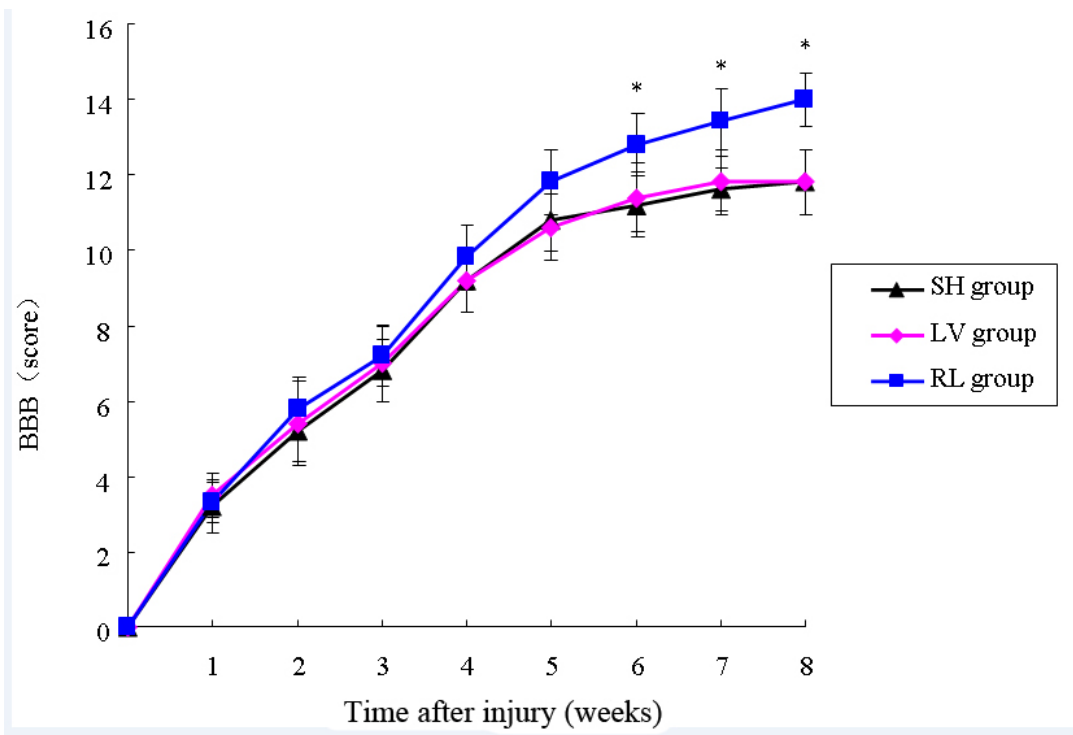

Figure 3. Behavior was evaluated with Basso-Beattie-Bresnahan (BBB) every week after spinal cord injury. For abbreviations, see legend to Figure 2. $* \mathrm{P}<0.05$. 


\section{Tract tracing}

A novel population of BDA-labeled axons could be observed extending past the lesion in the RL group, while only a few could be observed in the SH and LV groups (Figure 4).

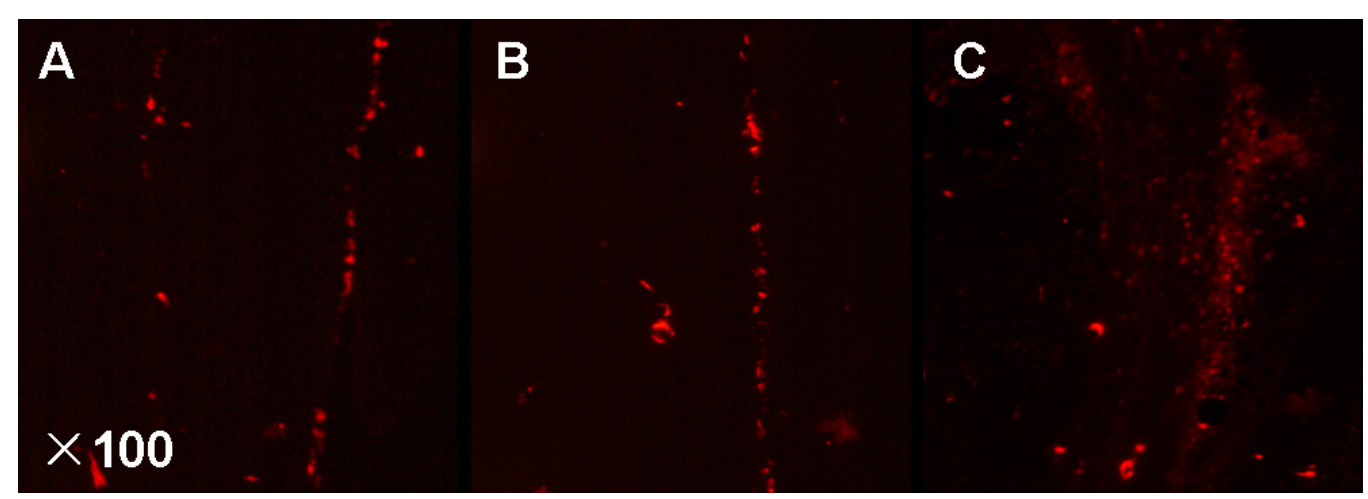

Figure 4. Axons in the lesion of spinal cord (100X). A. Sham-operated group; B. lentivirus vector group; C. recombinant lentivirus group.

\section{DISCUSSION}

In a recent study of Puram and Bonni (2011), APC-Cdh1 were not only found to be involved in regulating a variety of important biological processes, including cell growth, differentiation, matrix production, and apoptosis, but were also shown to play an important role in the nervous system. The APC-Cdh1 mediates the SnoN protein to regulate cell-intrinsic axonal morphogenesis (Matz, 1995; Topsakal et al., 2003; Lasorella et al, 2006; Stegmüller et al., 2008; Yang and Yang, 2012). Results of the present study indicated that the expression of Cdh1 mRNA in the operation group was significantly higher than that of the normal group, which, along with results of previous study, suggest that Cdh1 may act to stop axonal regeneration in spinal cord injuries.

BDA-TR is a highly sensitive tool for anterograde and retrograde pathway tracing studies of the nervous system. BDA-TR can be visualized along with fluorescent dextran amines using fluorescence labeling specific for BDA. In this study, we used four injection sites at the cortical surface, and after 2 weeks, BDA-TR was found to have reached lumbar vertebrae 1, and the axons in the lesion of spinal cord could be clearly seen (Choi et al., 2008; Harmey et al., 2009; Robbins and Cross, 2010; Yang et al., 2010; Hu et al., 2011; Puram and Bonni, 2011). Furthermore, motor behavior was assessed with the BBB test, which can reflect axonal regeneration and functional recovery in spinal cord injuries. We found that downregulating the expression of Cdh1 mRNA in the developing rat cerebellum in vivo specifically enhanced axonal growth.

We chose to perform injections in the sensorimotor cortex for several reasons. First, the neuron bodies of corticospinal tract innervation of hind limbs concentrate at the sensorimotor cortex of the anterior fontanelle, and APC-Cdh1 is expressed in these neurons. Second, soma of neurons of the sensorimotor cortex is very important for protection and the repair function 
of white matter. Third, a complex succession of changes takes place after spinal cord injury. At the early stage following injury, histopathological changes, bleeding, and inflammation result in an abundance of poisonous cytokines, oxy-radicals, and inflammatory cells in the microenvironment (Kim and Bonni, 2007; Yao et al., 2010; Yang et al., 2010). Therefore, the injection site of spinal cord injury may affect the function of siRNA. Finally, injection in the sensorimotor cortex allows direct action on the bodies of neurons.

In conclusion, the expression of Cdh1 mRNA in the sensorimotor cortex was higher in rats with spinal cord injuries, and inhibiting the expression of Cdh1 enhanced axon growth. Therefore, Cdh1 may represent a critical mechanism regulating axonal growth in the nervous system.

\section{REFERENCES}

Choi E, Dial JM, Jeong DE and Hall MC (2008). Unique D box and KEN box sequences limit ubiquitination of Acm1 and promote pseudosubstrate inhibition of the anaphase-promoting complex. J. Biol. Chem. 283: 23701-23710.

Harmey D, Smith A, Simanski S, Moussa CZ, et al. (2009). The anaphase promoting complex induces substrate degradation during neuronal differentiation. J. Biol. Chem. 284: 4317-4323.

Hu D, Qiao X, Wu G and Wan Y (2011). The emerging role of APC/CCdh1 in development. Semin. Cell Dev. Biol. 22: 579-585.

Kim AH and Bonni A (2007). Thinking within the D box: initial identification of Cdh1-APC substrates in the nervous system. Mol. Cell Neurosci. 34: 281-287.

Konishi Y, Stegmüller J, Matsuda T, Bonni Z, et al. (2004). Cdh1-A PC controls axonal growth and patterning in the mammalian brain. Science 303: 1026-1030.

Lasorella A, Stegmuller J, Guardavaccaro D, Liu G, et al. (2006). Degradation of Id2 by the anaphase-promoting complex couples cell cycle exit and axonal growth. Nature 442: 471-474.

Matz SP (1995). Connections of the olfactory bulb in the chinook salmon (Oncorhynchus tshawytscha). Brain Behav. Evol. 46: 108-120.

Puram SV and Bonni A (2011). Novel functions for the anaphase-promoting complex in neurobiology. Semin. Cell Dev. Biol. 22: 586-594.

Robbins JA and Cross FR (2010). Requirements and reasons for effective inhibition of the anaphase promoting complex activator CDH1. Mol. Biol. Cell 21: 914-925.

Schmittgen TD and Livak KJ (2008). Analyzing real-time PCR data by the comparative C(T) method. Nat. Protoc. 3: 1101-1108.

Stegmüller J, Konishi Y, Huynh MA, Yuan Z, et al. (2006). Cell-intrinsic regulation of axonal morphogenesis by the Cdh1-APC target SnoN. Neuron 50: 389-400.

Stegmüller J, Huynh MA, Yuan Z, Konishi Y, et al. (2008). TGFbeta-Smad2 signaling regulates the Cdh1-APC/SnoN pathway of axonal morphogenesis. J. Neurosci. 28: 1961-1969.

Topsakal C, Kilic N, Ozveren F, Akdemir I, et al. (2003). Effects of prostaglandin E1, melatonin, and oxytetracycline on lipid peroxidation, antioxidant defense system, paraoxonase (PON1) activities, and homocysteine levels in an animal model of spinal cord injury. Spine 28: 1643-1652.

Vogel G (2004). Nobel Prizes. Gold medal from cellular trash. Science 306: 400-401.

Wang YF, Lu G, Xu WB, Jin Z, et al. (2008). Effects of cryopreserved neural stem cells transplantation on rat axonal regeneration after spinal cord injury. Zhongguo Gu. Shang 21: 427-429.

Yang P and Yang Z (2012). Enhancing intrinsic growth capacity promotes adult CNS regeneration. J. Neurol. Sci. 312: 1-6.

Yang Y, Kim AH and Bonni A (2010). The dynamic ubiquitin ligase duo: Cdh1-APC and Cdc20-APC regulate neuronal morphogenesis and connectivity. Curr. Opin. Neurobiol. 20: 92-99.

Yao W, Qian W, Zhu C, Gui L, et al. (2010). Cdh1-APC is involved in the differentiation of neural stem cells into neurons. Neuroreport 21: 39-44. 